\title{
Efecto de las áreas de protección especial en la comunidad de macroinvertebrados de los ríos de la cuenca alta del Manzanares (Sierra de Guadarrama, Madrid)
}

\author{
Carmen Casado*, Laura Losada y Salvador Mollá
}

Departamento de Ecología. Facultad de Ciencias. Universidad Autónoma de Madrid. C/Darwin 2. Cantoblanco 28049. Madrid.

* Autor responsable de la correspondencia: c.casado@uam.es

Recibido: $28 / 9 / 10$

Aceptado: 23/3/11

\begin{abstract}
Effect of special protected areas in the macroinvertebrate community in the Manzanares upper basin (Sierra de Guadarrama, Madrid)

This contribution focuses on studying the quality and diversity of the three main rivers of the Upper Manzanares basin in Central Spain: the Samburiel-Navacerrada River, the Mediano River, and the Manzanares River. The main objective is the diagnosis of it's alterated state and to evaluate the current deterioration of the biological diversity of macroinvertebrates. To this effect a sampling was carried out during the most critical time of year for the system, summer of 2001.

Despite the fact that the physical environment is rather homogeneous in the whole area, there are differences in the characteristics and functional features of the streams. Those differences seem mainly related to land-use and water management, factors themselves dependent on whether the sub-basin is located inside or outside the protected area of 'Cuenca Alta del Manzanares' Regional Park (PRCAM). The Manzanares river is the one presenting the highest state of conservation and water quality, with high values for richness, diversity, and biotic indexes. The Samburiel river, on the other hand, is the one presenting the worst conditions and the greatest degree of deterioration, due to flow regulation as well as pollution. It's macroinvertebrate communities are extremely impoverished and present high spatial heterogeneity. The Mediano stream shows an intermediate condition, partly due to its artificial temporary flow (water withdrawals in its middle reach). This explains and the fact that the macroinvertebrate communities have low-density populations, with diminishing species richness as well as water quality.
\end{abstract}

Key words: Headwater mountain streams, water quality, macroinvertebrates, diversity, IBMWP, protected areas.

\section{RESUMEN}

Efecto de las áreas de protección especial en la comunidad de macroinvertebrados de los ríos de la cuenca alta del Manzanares (Sierra de Guadarrama, Madrid)

El presente trabajo se centra en el estudio de la calidad y diversidad de los tres principales ríos de la Cuenca Alta del río Manzanares: el río Samburiel-Navacerrada, el río Manzanares y el arroyo Mediano. El objetivo primordial es el diagnóstico de su estado de alteración y la valoración de la actual situación de deterioro de la diversidad biológica de macroinvertebrados. Para ello se realizó un muestreo durante la época más crítica del sistema, verano de 2001.

A pesar de que el medio físico de las tres subcuencas es relativamente homogéneo, existen divergencias en las características y funcionamiento de sus cauces que se deben básicamente a los distintos usos que el hombre está haciendo de cada una de las cuencas y del agua, muy relacionados con su localización dentro o fuera del área protegida del Parque Regional de la Cuenca Alta del Manzanares (PRCAM). El río Manzanares es el que presenta un mejor estado de conservación y calidad de sus aguas, con elevados valores de riqueza, diversidad e índices bióticos. El río Samburiel es, por el contrario, el que presenta peores condiciones y un mayor grado de deterioro, tanto por regulación de caudales como por contaminación. Las comunidades de macroinvertebrados están muy empobrecidas y presentan fuertes variaciones espaciales. El arroyo Mediano muestra una situación intermedia, debido en parte a su artificial temporalidad (por detracción de caudal en su tramo medio). Por ello no es de extrañar que las poblaciones de macroinvertebrados sean poco densas, disminuyendo los valores de riqueza taxonómica y de calidad del agua.

Palabras clave: Arroyos de montaña, calidad del agua, macroinvertebrados, diversidad, IBMWP, áreas protegidas. 


\section{INTRODUCCIÓN}

En la Península Ibérica los principales centros de reserva de la diversidad biológica son las áreas de montaña, en especial sus altitudes medias y altas. El uso tradicional en los ambientes mediterráneos ha sido la explotación de alta intensidad en las zonas bajas, principalmente con fines agrícolas, lo que permitió a las áreas más agrestes (zonas elevadas y de mayor pendiente) conservar un alto grado de naturalidad y mantener un elevado índice de riqueza de especies hasta nuestros días. Esto, junto con su contribución al control de la erosión y a la captación de agua, ha originado que en la actualidad el $73 \%$ del territorio español por encima de 1500 metros y el $18 \%$ de la superficie comprendida entre 1000 y 1500 metros se encuentren protegidos bajo alguna de las más de 40 figuras distintas de protección de espacios naturales que se utilizan en el conjunto del estado (Europarc-España, 2010).

Esta situación la encontramos reflejada en la Comunidad Autónoma de Madrid (CAM), donde los puntos de mayor densidad de especies (hot spots) se localizan preferentemente en la Sierra de Guadarrama, tanto en zonas medias como altas. Sin embargo, en la última década los pequeños núcleos de población de la sierra madrileña han experimentado un desmesurado crecimiento urbanístico debido a la especulación del suelo. Se han transformado grandes extensiones serranas en áreas urbanizadas, con la consiguiente expansión de las infraestructuras asociadas (viarias, hidráulicas, de servicios, etc.). Esta serie de cambios de uso del territorio ha originado en las redes fluviales unas alteraciones que vienen a sumarse a las ya sufridas desde hace décadas por los clásicos y tradicionales usos recreativos de la población de Madrid y áreas metropolitanas. La protección de estos ecosistemas es fundamental para la conservación de su biodiversidad (Abell, 2002; Saunders et al., 2002.), ya que con la alteración de su estructura y contaminación del agua, los ríos han perdido gran parte de la diversidad biológica (Malmqvist \& Rundle, 2002; Casas et al., 2006; Prenda et al., 2006; Benetti et al., 2007). El estudio de los sistemas fluviales permite valorar las consecuencias de todos estos cambios de usos del territorio al detectar las alteraciones que los mismos originan en la cantidad y calidad de sus aguas, en las comunidades que albergan y en los procesos (físicos, químicos y biológicos) que se desarrollan en el cauce y riberas fluviales.

En la vertiente suroeste de la Sierra de Guadarrama se localiza la red fluvial del río Manzanares, gran parte de la cual está incluida dentro del actual Parque Regional de la Cuenca Alta del río Manzanares (PRCAM, 1985) declarado Reserva de la Biosfera por la UNESCO en 1993. En la actualidad se encuentra abierto el procedimiento para declararlo Parque Nacional de la Sierra de Guadarrama por iniciativa de las Comunidades Autónomas de Castilla y León y de Madrid (cuya propuesta inicial ha sido por fin aprobada por el gobierno regional), ampliando así tanto la figura como el área de protección. Sin embargo, esto no garantiza la protección de las comunidades lóticas (Mancini et al., 2005) ni la conservación de la biodiversidad (Abel et al., 2007) ya que la declaración legal de un espacio protegido y el desarrollo de los instrumentos de planificación son condición necesaria pero no suficiente (EuroparcEspaña, 2010), especialmente para los hábitats y especies acuáticas (Saunders et al., 2002).

A pesar de la importancia de los ríos en la vertebración del territorio del PRCAM y de los numerosos estudios realizados en este parque, la mayoría de los trabajos ignoran a los ecosistemas fluviales, de los que solo se han llevado a cabo estudios puntuales e inconexos. La primera publicación existente sobre el río Manzanares data de principios del siglo XX, se trata de una monografía que constituye el primer estudio de ecología fluvial en España y uno de los primeros europeos (Vélaz de Medrano y Ugarte, 1933), en la que se recogen datos físico-químicos y de las comunidades de peces. En esta misma década también encontramos algunas referencias descriptivas sobre algas y plancton del río Manzanares y en la década de los 60, algún informe sobre la contaminación química y microbiológica, de escasa difusión. No es hasta la década de los 80 cuando empezamos a encontrar las primeras publicaciones y monografías. Cubillo en 1985 realiza un informe sobre la calidad del agua de los principales 
ríos de Madrid, entre ellos el Manzanares. García de Jalón y Gónzález del Tánago (1986) describen y aplican diversos índices biológicos de calidad del agua a la parte alta del río Manzanares. Monzón (1986) describe la organización longitudinal de las comunidades de macroinvertebrados bentónicos fluviales del eje principal del río Manzanares. Montes et al. (1987) realizan un estudio integral de las riberas y el cauce fluvial del tramo comprendido entre los embalses de Santillana y El Pardo, que pretendió sentar las bases para una propuesta de recuperación ecológica del tramo medio del río Manzanares a través de un mejor uso y gestión de la cuenca vertiente. En $1990 \mathrm{Cu}$ billo et al., publican una monografía centrada en una propuesta de gestión de los caudales ecológicos de los ríos de la CAM en la que se incluye al río Manzanares. En 1991 Monzón et al., en un estudio realizado en el tramo alto del río Manzanares y el arroyo Mediano, describen la variación longitudinal de la calidad del agua (índice BMWP'), la composición y estructura de las comunidades de macroinvertebrados acuáticos y su estructura trófica correlacionándola con los patrones encontrados de las distintas fracciones de materia orgánica. Gómez-Limón y García Avilés (1992) realizan un breve estudio acerca del impacto de las actividades recreativas en el río Manzanares y arroyo de la Majadilla (núcleo central del parque) utilizando para ello el índice biótico de calidad del agua BMWP'. Más recientemente, Serrano (2000) y Serrano et al. (2004) describen las comunidades epilíticas de cianobacterias en el arroyo Mediano, Gutiérrez Teira (2003), las comunidades de macroinvertebrados y los ciclos vitales y estimas de producción secundaria para algunas especies en un tramo de cabecera del río Manzanares, Pérez Siles (2004), la dinámica poblacional y producción secundaria de Epeorus torrentium en el arroyo Mediano, y Losada (2004), las comunidades de macroinvertebrados de la cuenca alta del Manzanares.

Como se puede apreciar, la información existente carece de una continuidad en el tiempo y, junto con la falta de información reciente, no permite valorar la situación actual de los ecosistemas fluviales de la vertiente sur de la Sierra de Guadarrama, ni siquiera dentro del PRCAM, lo que pone de manifiesto la poca atención que han recibido las masas o corrientes de agua epicontinental en áreas protegidas (Gilmar., 2004; Prenda et al., 2006; Abell et al., 2007) y la necesidad de prestar mayor atención tanto a la conservación de la biodiversidad acuática continental en áreas formalmente protegidas (Mancini et al., 2005, Nel et al., 2007, Europarc-España, 2010) como a las estrategias de diseño y gestión de áreas acuáticas continentales protegidas (Saunders et al., 2002; Nel et al., 2007).

El efecto de la protección de espacios en áreas naturales y/o periurbanas sobre los ecosistemas fluviales o sobre su fauna de macroinvertebrados ha sido también poco estudiado en la península ibérica, destacando los trabajos de Rieradevall et al. (1999) y Bonada et al. (2000).

El objetivo principal de este trabajo es evaluar la situación actual de calidad y diversidad biológica de los tres principales sistemas fluviales de la cuenca alta del río Manzanares, contrastar su estado de alteración y deterioro con los distintos tipos de actividades y usos que el hombre está haciendo de cada una de las cuencas y del agua, muy relacionados con su localización dentro o fuera del área protegida del PRCAM. Ello nos permite verificar el papel o contribución que las áreas protegidas pueden tener en la conservación de la diversidad biológica y los ecosistemas fluviales de la Sierra de Guadarrama, partiendo de la hipótesis de que estas áreas benefician a la fauna y calidad biológica fluvial, por lo que esperamos encontrar mejores condiciones en las estaciones y tramos fluviales ubicados dentro del parque.

\section{ÁREA DE ESTUDIO}

La cuenca alta del río Manzanares se encuentra drenada por tres sistemas fluviales, que de oeste a este son: el río Samburiel y sus dos principales afluentes Angostura y Gargantilla, el río Manzanares y su principal afluente, el Majadilla, y el arroyo Mediano (Fig. 1). Las tres subcuencas estudiadas drenan un área de $161.1 \mathrm{~km}^{2}\left(90.9 \mathrm{~km}^{2}\right.$, $49.4 \mathrm{~km}^{2}$, y $20.8 \mathrm{~km}^{2}$ respectivamente).

El sustrato de las tres cuencas está constituido en su mayoría por materiales hercínicos áci- 


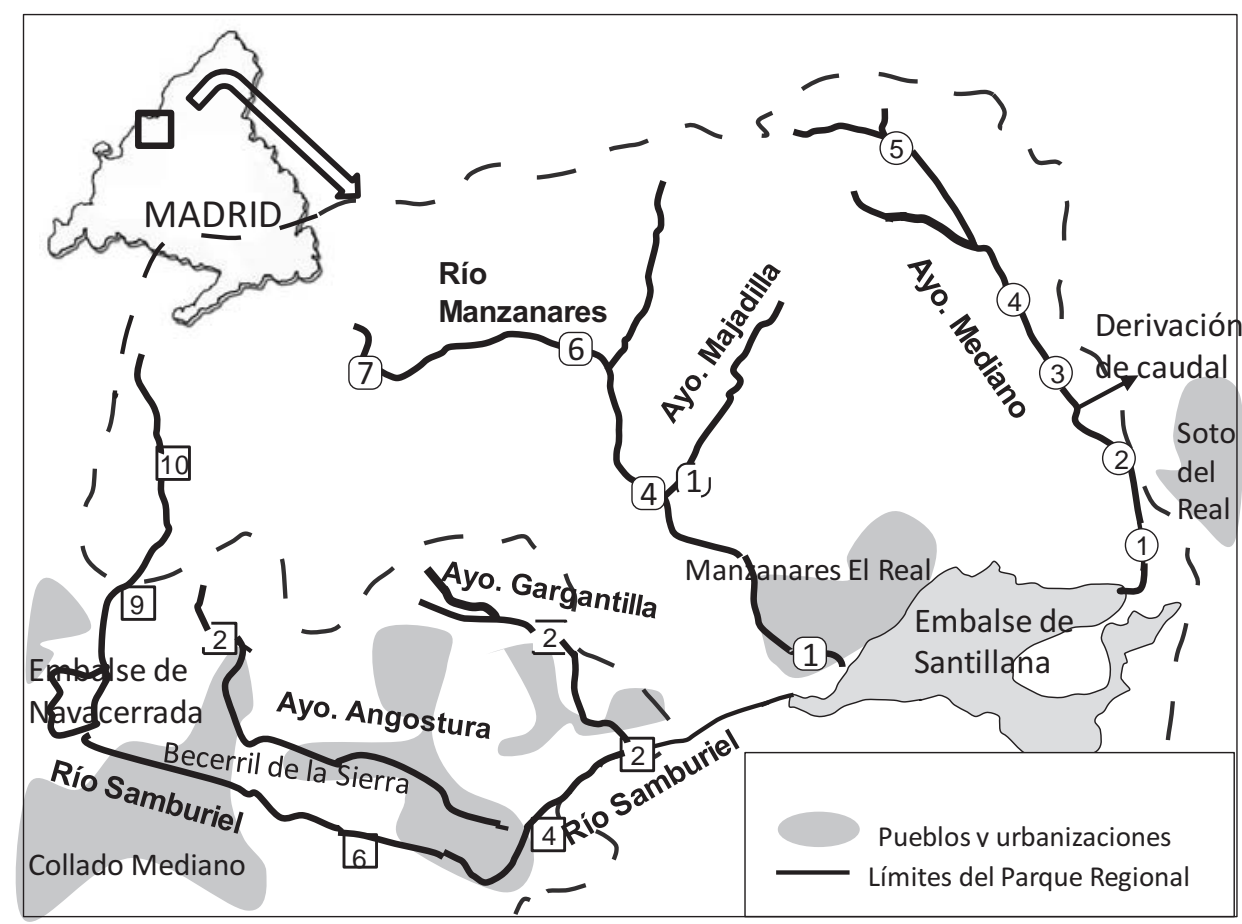

Figura 1. Localización del área de estudio y estaciones de muestreo. Location of the study area and sampling sites.

dos (granitos y granodiorítas) con afloramientos metamórficos de gneises. En los tramos bajos, a la altura del embalse de Santillana, donde confluyen los tres ríos, aparecen materiales aluviales cuaternarios y una pequeña mancha de dolomías cretácicas con incrustaciones margosas en la cuenca del Samburiel. El clima es de tipo mediterráneo templado-húmedo, con una media de precipitación anual entre 1100 y $900 \mathrm{~mm}$. La vegetación de la cuenca se ordena en el gradiente altitudinal típico de la vertiente sur de la Sierra de Guadarrama (Blanco et al., 1997), pastos de montaña, piornales y enebrales en las cotas altitudinales superiores $(>2000 \mathrm{~m})$, pinares (2000$1200 \mathrm{~m})$, melojares (1200-1000 m) y encinares adehesados $(<1000 \mathrm{~m})$. El régimen hidrológico natural de estos ríos y arroyos es de tipo pluvionival, aunque está muy modificado artificialmente por embalses y represas destinadas a almacenamiento de agua para abastecimiento y ganadería.

Sin embargo, las actividades y usos humanos de las tres cuencas colindantes difieren notablemente. Únicamente la totalidad de la cuenca del Manzanares, la mayor parte de la del Mediano y algún tramo aislado de la cabecera del Samburiel, se encuentran incluidas en el PRCAM (Fig. 1), por lo que gozan de mayor protección frente a la especulación urbanística que sufre este sector de la Sierra de Guadarrama, así como frente a las tradicionales actividades derivadas de la ganadería y el esparcimiento. Por este motivo, el sistema más alterado es el del Samburiel, que además atraviesa varios núcleos urbanos de importancia. El Manzanares discurre en la zona de cabecera por áreas poco accesibles, de tráfico restringido y altamente protegidas, pero en su tramo medio sufre los efectos de una alta afluencia de visitantes, y algo más abajo discurre por las urbanizaciones del entorno de Manzanares el Real. La cuenca del Mediano se encuentra libre de áreas recreativas y núcleos de población hasta casi el final de su recorrido, pero en su tramo medio sufre una detracción de su caudal, que es derivado y canalizado hasta el pequeño embalse de Los Palancares, que sirve para abastecer a la población de Soto del Real. Aguas abajo de la toma, el arroyo se convierte en un cauce temporal ya que extraen total o parcialmente (según la época del año) el caudal circulante. 


\section{METODOLOGÍA}

A partir de información cartográfica sobre la topografía, litología, vegetación y usos del suelo, se establecieron un total de 31 estaciones de muestreo, pero al realizarse la campaña de campo durante junio de 2001, 15 se encontraron totalmente secas o eran pequeños charcos de agua de lluvia, dando como resultado un total de 17 puntos repartidos del siguiente modo: 7 en el sistema fluvial del Manzanares, 5 en el del arroyo Mediano y 5 en el del río Samburiel. (Su localización y principales características se recogen en la Fig. 1 y Tabla 1).

En cada punto de muestreo se registró in situ la temperatura del agua, el $\mathrm{pH}$, la conductividad eléctrica y la concentración de oxígeno disuelto mediante los correspondientes aparatos de campo (CrisonPH25, WTWLF320, WTWOxi320). Además, se recogieron muestras de agua por triplicado para el análisis in situ de alcalinidad y nutrientes (nitrato, nitrito, amonio y fósforo reactivo soluble); con un espectrofotómetro portátil HachDR/2010 se determinaron el resto de los iones mayoritarios (sulfatos, cloruros, calcio, magnesio, sodio y potasio) a partir de muestras conservadas a $4{ }^{\circ} \mathrm{C}$, hasta que se procedió a su análisis en laboratorio mediante análisis iónico capilar (Waters capillary for analyser, 1995). La determinación de la composición iónica de las aguas se realiza en base al cálculo del porcentaje relativo de equivalentes químicos que aportan cada uno de los distintos iones mayoritarios dispuestos por orden de dominancia en un balance iónico (Diagramas triangulares de series iónicas de Eugster \& Hardie, 1987).

Para la captura y cuantificación de macroinvertebrados acuáticos se utilizó el método de captura por unidad de esfuerzo (5 minutos de tiempo efectivo de mangueo), empleando una red de mano cuadrada de $33 \mathrm{~cm}$ de lado y de $250 \mu \mathrm{m}$ de luz de malla (Torralba y Ocharan, 2007). Las muestras obtenidas fueron preservadas fijándolas con formol al $4 \%$ y trasladadas al laboratorio para su separación, identificación, recuento y posterior conservación en alcohol al $70 \%$. Se han empleado tres parámetros para valorar la estructura de la comunidad, riqueza taxonómica $\left(\mathrm{n} .{ }^{\circ} \mathrm{de}\right.$ taxones/estación), abundancia (n. ${ }^{\circ}$ de individuos/ estación) y diversidad (índice de Shannon-Wie-

Tabla 1. Localización de las estaciones de muestreo, códigos y valores medios de los principales parámetros físico-químicos y nutrientes analizados en las aguas de los ríos estudiados. Location of sampling sites, codes and mean values of the main physicalchemical and nutrient parameters of the studied streams.

\begin{tabular}{|c|c|c|c|c|c|c|c|c|c|c|c|c|c|c|}
\hline Estación & Código & $\begin{array}{c}\text { Coordenadas } \\
\text { UTM }\end{array}$ & $\begin{array}{c}\text { Altitud } \\
\text { m }\end{array}$ & $\begin{array}{c}\text { Anchura } \\
\text { m }\end{array}$ & $\begin{array}{l}\text { Profundidad } \\
\text { cm }\end{array}$ & $\begin{array}{c}\text { C. E. } \\
\mu S / \mathrm{cm}\end{array}$ & $\begin{array}{c}\mathrm{O} 2 \\
\mathrm{mg} / \mathrm{l}\end{array}$ & $\begin{array}{c}\mathbf{T}^{\mathbf{a}} \text { agua } \\
{ }^{\circ} \mathbf{C}\end{array}$ & $\begin{array}{l}\text { Sat } \mathrm{O}_{2} \\
\%\end{array}$ & $\mathbf{p H}$ & $\begin{array}{l}\mathrm{NO}_{3}^{-} \\
\mathrm{mg} / \mathrm{l}\end{array}$ & $\begin{array}{l}\mathrm{NO}_{2}^{-} \\
\mathrm{mg} / \mathrm{l}\end{array}$ & $\begin{array}{l}\mathrm{NH}_{4}^{+} \\
\mathrm{mg} / \mathrm{l}\end{array}$ & $\begin{array}{l}\mathrm{PO}_{4}^{3-} \\
\mathrm{mg} / \mathrm{l}\end{array}$ \\
\hline \multicolumn{15}{|c|}{ Cuenca del arroyo Mediano } \\
\hline Mediano 5 & Med 5 & 30TVL285173 & 1810 & 1.6 & 40 & 13 & 9.58 & 9.5 & 97.8 & 6.8 & 0.27 & 0.13 & 0.06 & 0.49 \\
\hline Mediano 4 & Med 4 & 30TVL297156 & 1320 & 1.3 & 40 & 16 & 9.35 & 11.4 & 97.3 & 6.6 & 0.40 & 0.07 & 0.03 & 0.07 \\
\hline Mediano 3 & Med 3 & 30TVL311133 & 1095 & 1.2 & $40-60$ & 29 & 8.18 & 16.9 & 95.3 & 6.8 & 0.40 & 0.09 & 0.11 & 1.62 \\
\hline Mediano 2 & Med 2 & 30TVL323120 & 935 & $3-5$ & 15 & 42 & 7.10 & 15.6 & 79.3 & 6.8 & 0.04 & 0.09 & 0.04 & 0.43 \\
\hline Mediano 1 & Med 1 & 30TVL324106 & 905 & 3 & $5-12$ & 106 & 7.22 & 15.6 & 80.5 & 7.2 & 0.47 & 0.08 & 0.04 & 3.20 \\
\hline \multicolumn{15}{|c|}{ Cuenca de río Manzanares } \\
\hline Manzanares 7 & Man 7 & 30TVL209137 & 1740 & 3.7 & 30 & 9 & 9.14 & 10.2 & 99.9 & 6.6 & 0.53 & 0.09 & 0.05 & 2.00 \\
\hline Manzanares 6 & Man 6 & 30TVL232140 & 1200 & 6 & $40-60$ & 12 & 8.98 & 12.9 & 98.0 & 6.6 & 0.50 & 0.07 & 0.12 & 1.85 \\
\hline Manzanares 4 & Man 4 & 30TVL246116 & 1030 & 6 & 60 & 15 & 9.42 & 12.1 & 97.9 & 6.8 & 0.44 & 0.10 & 0.18 & 4.20 \\
\hline Manzanares 1 & Man 1 & 30TVL270087 & 910 & 7 & 60 & 21 & 8.48 & 15.7 & 94.0 & 6.7 & 0.41 & 0.09 & 0.06 & 8.70 \\
\hline Majadilla & Maj 1 & 30TVL247117 & 1025 & $2-4$ & $5-10$ & 20 & 8.84 & 12.0 & 91.7 & 6.8 & 0.43 & 0.11 & 0.03 & 1.03 \\
\hline \multicolumn{15}{|c|}{ Cuenca del río Samburiel } \\
\hline Samburiel 10 & Sam 10 & 30TVL164114 & 1330 & 1.8 & 14 & 22 & 8.40 & 16.2 & 100.2 & 7.2 & 0.38 & 0.06 & 0.02 & 0.14 \\
\hline Samburiel 9 & Sam 9 & 30TVL156094 & 1200 & $5-6$ & 10 & 25 & 8.54 & 18.2 & 106.3 & 7.2 & 0.38 & 0.10 & 0.01 & 0.19 \\
\hline Samburiel 6 & Sam 6 & 30TVL191062 & 970 & $2-3$ & $5-10$ & 120 & 9.40 & 18.1 & 111.4 & 7.5 & 0.70 & 0.13 & 0.27 & 0.33 \\
\hline Samburiel 4 & Sam 4 & 30TVL221058 & 940 & 4 & 20 & 173 & 8.03 & 17.4 & $94 .$. & 7.5 & 0.48 & 0.13 & 0.05 & 0.16 \\
\hline Samburiel 2 & Sam 2 & 30TVL237075 & 910 & $4-7$ & $5-7$ & 198 & 8.96 & 16.6 & 102.7 & 7.7 & 0.45 & 0.15 & 0.04 & 0.29 \\
\hline Angostura & Ang 2 & 30TVL173091 & 1100 & 0.2 & 80 & 56 & 8.50 & 15.9 & 98. & 6.8 & 0.34 & 0.09 & $<0.01$ & 0.17 \\
\hline Gargantilla & Gar 2 & 30TVL207096 & 960 & $1-2$ & 24 & 105 & 9.76 & 16.5 & 111.0 & 7.2 & 0.45 & 0.17 & 0.03 & 0.42 \\
\hline
\end{tabular}


ners, utilizando $\log _{2}$ ). Como índice biótico de calidad del agua se ha utilizado el índice IBMWP (Alba-Tercedor et al., 2002; Bonada et al., 2002).

Los análisis estadísticos que se realizaron con los datos fueron de clasificación y ordenación, utilizando el paquete estadístico Pc-Ord v.4. El dendrograma ha sido construido por un análisis de clúster (UPGMA), utilizándose el índice de Jaccard. La matriz de datos se construyó con la presencia o ausencia de las familias en cada punto (Benetti et al., 2007). En la ordenación se empleó el análisis escalado multidimensional no paramétrico (non-metric multidimensional scaling) adecuado para datos que no son normales, continuos o con gran cantidad de ceros, para los cuales la aplicación de otros estadísticos como el ACP resulta inadecuada (Leska \& Karr, 1996).

\section{RESULTADOS}

\section{Características físicas y químicas del agua}

En la tabla 1 se observa que todas las estaciones tienen una alta saturación de oxígeno (superior al $80 \%$ ), baja mineralización (conductividad eléctrica inferior a $200 \mu \mathrm{S} / \mathrm{cm}$ ) y $\mathrm{pH}$ entre 6.5-7.7.
En todas las subcuencas se observa el clásico aumento de la mineralización a lo largo del eje cabecera-desembocadura, aunque podemos diferenciar claramente dos grados de concentración de sales en disolución. Aguas con una mineralización muy baja, como las del río Manzanares y su arroyo Majadilla (conductividad: 9-21 $\mathrm{S} / \mathrm{cm}$ ), las del tramo alto del arroyo Mediano antes de la detracción de sus caudales (13-29 $\mu \mathrm{S} / \mathrm{cm})$ y las dos estaciones de nacimiento del río Samburiel localizadas en el denominado arroyo Navacerrada $(22-25 \mu \mathrm{S} / \mathrm{cm})$. El grupo de aguas que podríamos clasificar como de mediabaja mineralización incluye todas las estaciones del río Samburiel, desde la salida del embalse de Navacerrada hasta la cola del embalse de Santillana y sus afluentes $(120-198 \mu \mathrm{S} / \mathrm{cm})$, y el tramo bajo del arroyo Mediano después de la captación y canalización de su caudal hacia el embalse de los Palancares (42-106 $\mu \mathrm{S} / \mathrm{cm})$.

Estas tendencias espaciales también son observables atendiendo a la composición iónica de las aguas de las tres subcuencas, diferenciándose claramente dos tipos de aguas. Estos resultados se representan gráficamente en el diagrama de la figura 2. Las aguas menos mineralizadas de las cuencas del río Manzanares y del tramo alto del

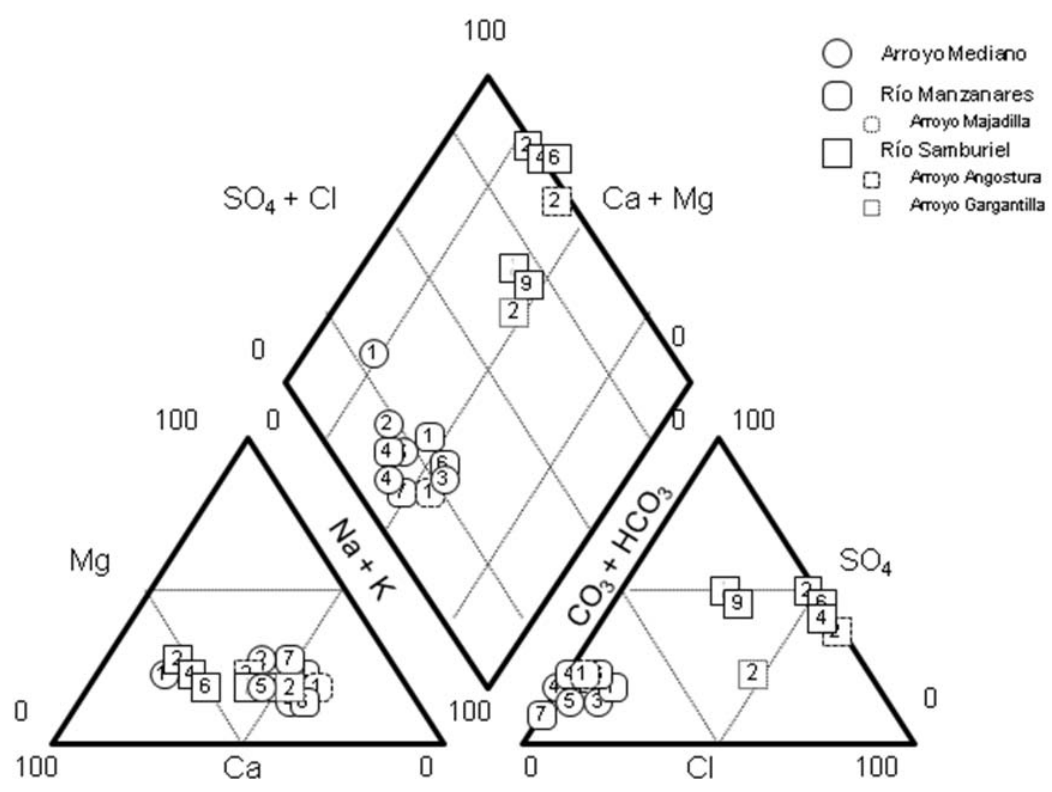

Figura 2. Diagrama de Piper de la composición iónica de los ríos estudiados. Piper diagram of the ionic composition of the studied streams. 
arroyo Mediano son de tipo bicarbonatado-sódicas, mientras que las de cabecera del Samburiel son sulfatado-sódicas. En las aguas de mayor mineralización, el calcio domina claramente sobre el sodio, así, las del río Samburiel en su tramo medio y bajo son de tipo clorurado cálcicas y las del tramo bajo del Mediano (donde el arroyo experimenta una desecación estival forzada de forma artificial) siguen siendo bicarbonatadas pero de carácter mixto cálcico-sódicas en la estación superior (Med2) y claramente bicarbonatado-cálcicas en la desembocadura (Med1).

Respecto a los nutrientes (Tabla 1), las concentraciones de las tres formas de nitrógeno inorgánico disuelto siempre son bajas. Los mayores valores los encontramos en el río Samburiel tras el embalse de Navacerrada (Sam6), en forma de nitrato y de amonio. También es en el tramo bajo de este río y en su arroyo Gargantilla donde observamos los máximos valores de nitritos, aunque nunca superan los $0.17 \mathrm{mg} / \mathrm{l}$. Otros valores relativamente elevados de amonio se presentan en Man6, Man4 y Med3, estaciones sometidas a una fuerte presión antrópica producida por la afluencia de visitantes.

Se detectan valores inesperadamente elevados de fosfatos en el río Manzanares (especialmente en su desembocadura), en su arroyo Majadilla y en el arroyo Mediano. Además, desconcertantemente la proporción con los valores de nitrógeno está muy descompensada.

\section{Composición, abundancia, riqueza y diversidad biológica de la comunidad de macroinvertebrados}

Se han identificado 22864 individuos, pertenecientes a 163 géneros de macroinvertebrados acuáticos agrupados en 85 familias. El listado completo de los taxones reconocidos se muestra en Losada (2004). Las familias mejor representadas por su frecuencia y abundancia son Baetidae (8805 individuos), Chironomidae (3701), Simuliidae (3588), Leptophlebiidae (1901), y en menor medida Leuctridae (682), Elmidae (620) y Perlodidae (269), aunque estas dos últimas apenas están representadas en el río Samburiel, donde por el contrario son importantes los Naididae (335) y Lum-

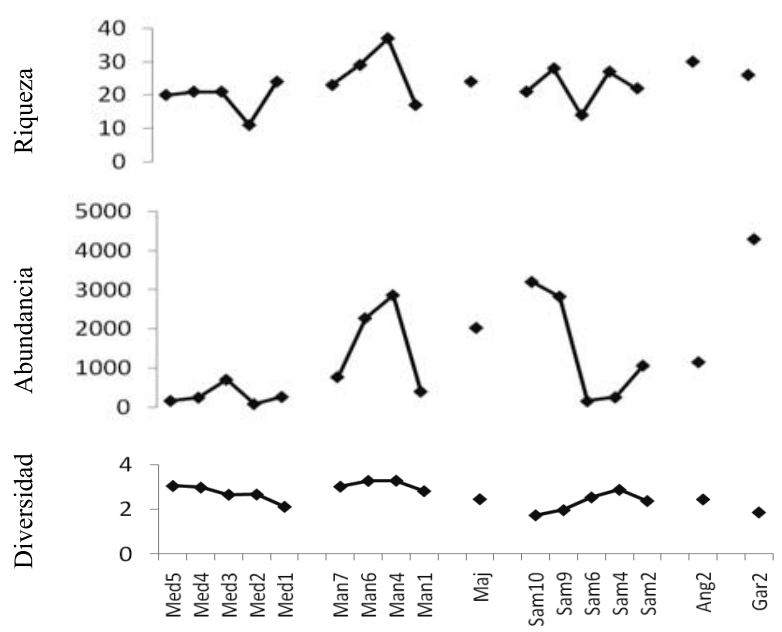

Figura 3. Valores de riqueza taxonómica, abundancia y diversidad de las comunidades de macroinvertebrados en los tres ríos estudiados. Values of taxonomic richness, abundance and diversity of macrobenthic communities at the three sampled rivers.

briculidae (208). Los restantes grupos tienen distribuciones más restringidas, con densidades muy inferiores o aparecen solo de forma ocasional.

El río Manzanares, localizado íntegramente dentro de la zona protegida del Parque al que da nombre, es el que presenta los máximos valores de riqueza taxonómica (17-37 taxones/estación) y diversidad biológica (Fig. 3), con valores ligeramente superiores a 3 bits ind $^{-1}$ propios de ríos bien conservados, excepto en el caso de la desembocadura (Man1 con 2.82). Se observa un patrón de evolución longitudinal en el que la riqueza, abundancia de macroinvertebrados y diversidad aumentan rápidamente desde la cabecera, alcanzando los valores máximos en el tramo medio (Man4), para disminuir bruscamente en la estación de desembocadura (Man1) al pasar por la población de Manzanares El Real.

A pesar de que los órdenes que aportan la mayor riqueza taxonómica son Trichoptera $(20 \%)$, Ephemeroptera (15\%), Diptera $(14 \%)$ y Plecóptera (13\%), la comunidad de bentos (Fig. 4) está dominada por Baetidae, Heptageniidae, Leptophlebiidae y Ephemerellidae (suponen casi el $49 \%$ de la abundancia) y Chironomidae y Simuliidae (cuya abundancia aumenta en los tramos bajos). También se puede observar una 


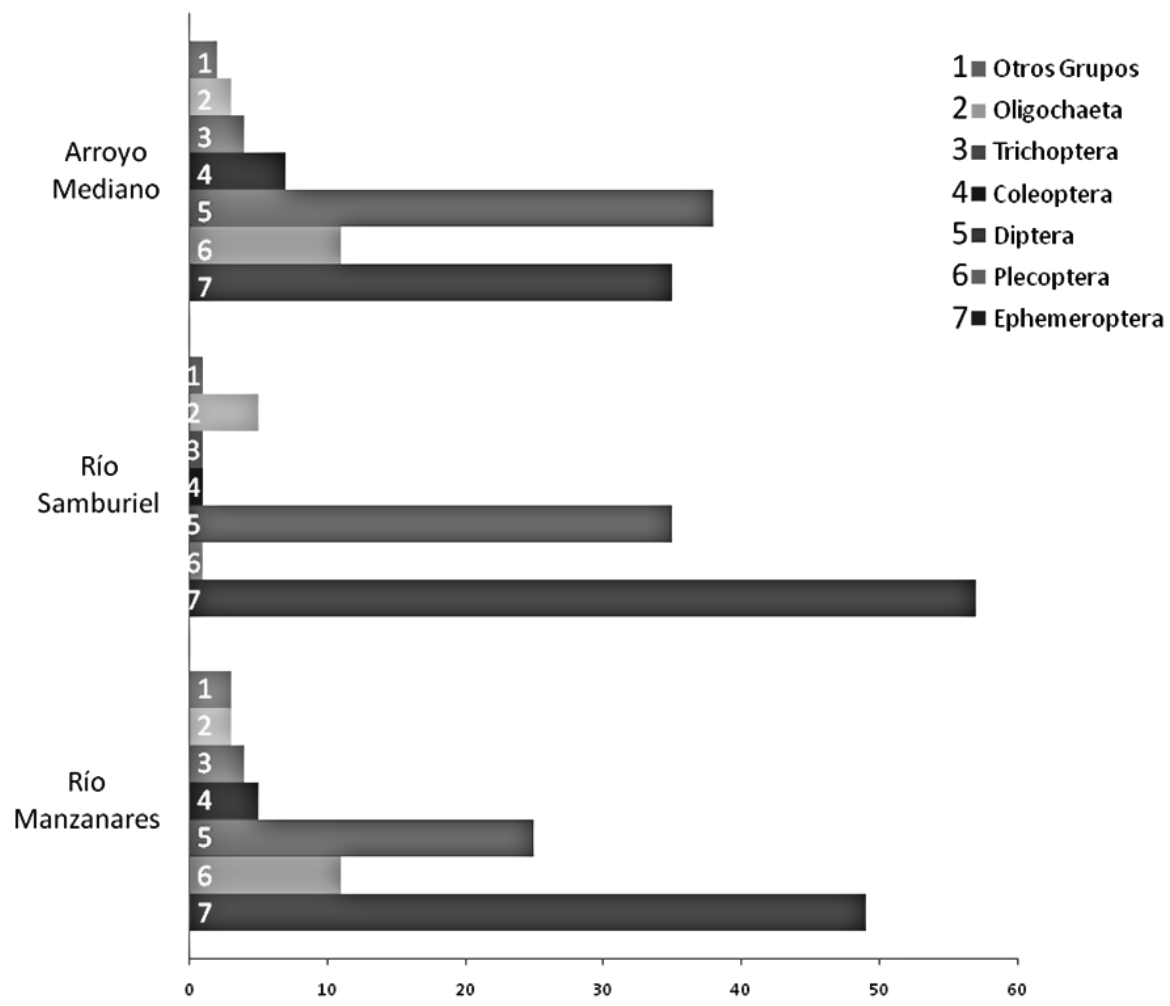

Figura 4. Composición porcentual de los órdenes más abundantes de macroinvertebrados en los tres ríos estudiados. Percentage of the most abundant orders of macroinvertebrates in the sampled streams.

cierta abundancia en cabecera de Elmidae acompañados de Helodidae y Dytiscidae, que disminuyen rápidamente hacia tramos inferiores donde es sustituido por Naididae. Es de destacar la presencia constante de Leuctridae, Perlodidae y en menor proporción Chloroperlidae, lo que hace referencia a una buena calidad de las aguas, ya que estos organismos son de los más exigentes en cuanto a condiciones ambientales. Rhyacophilidae, Hydropsychidae, Polycentropidae, Psychomyidae y Sericostomatidae siguen un patrón semejante de evolución aunque con menores abundancias.

El arroyo Majadilla, afluente del Manzanares, muestra un valor inferior del índice de diversidad, debido básicamente a que a pesar de tener una elevada riqueza, la comunidad se encuentra dominada por las poblaciones de Chironomidae, Simulidae, Baetidae y Leptophlebiidae que alcanzan unas elevadas abundancias poblacionales, manteniendo a los restantes grupos en unas proporciones prácticamente testimoniales.
El arroyo Mediano presenta los menores valores de riqueza (11-24 taxones/estación) y abundancia (97-714 individuos/estación) de los tres ríos, en parte esperable, ya que es la cuenca y el cauce de menor tamaño y desarrollo morfológico. Los valores de riqueza faunística son muy homogéneos (entorno a 20) excepto en el tramo medio-bajo (Med2) donde experimenta una caída drástica coincidiendo con varias alteraciones como detracción del caudal y canalización parcial del cauce. La aportación a la riqueza taxonómica está muy compartida entre Ephemeroptera (24\%), Coleoptera (18\%), Diptera (16\%), Plecoptera $(12 \%)$ y Trichoptera. La variación longitudinal de la abundancia sigue casi el mismo patrón que la riqueza faunística, las comunidades de cabecera presentan bajos valores (Med5 y Med4 con 177 y 259 individuos) que aumentan ligeramente aguas abajo (Med3 con 714 individuos), disminuyen bruscamente en el punto Med2 (97 individuos) y finalmente se recuperan de forma parcial en la desembocadura (Med1 con 278 individuos). La 
diversidad presenta valores intermedios (siempre superiores a 2.5 bits ind $^{-1}$ ) excepto en la última estación (Med1), modificada como área recreativa de Soto del Real, donde desciende a 2.12. El patrón de evolución longitudinal es inverso al observado en el río Manzanares, los valores más elevados se alcanzan en el nacimiento y disminuyen progresivamente de cabecera a desembocadura.

El río Samburiel muestra importantes oscilaciones en los valores de riqueza (14-30 taxones/estación) y especialmente de abundancia (167-4285 individuos/estación), que ponen de relieve las sucesivas alteraciones e impactos antrópicos y las recuperaciones del río, que es sin duda el más alterado de los tres. Se observa un aumento de la riqueza taxonómica desde cabecera con una disminución drástica en el tramo medio (Sam6, aguas abajo del embalse de Navacerrada). La mayor riqueza taxómica la aportan los Diptera (24\%), Coleoptera (21\%), Oligochaeta $(14 \%)$ y Ephemeroptera $(13 \%)$. En este río se recogen los valores máximos puntuales de abundancia de las tres cuencas. Las mayores abundancias se presentan atípicamente en la cabecera (Sam10 y Sam9 con 3199 y 2827 individuos respectivamente), en las siguientes estaciones disminuye drásticamente pasando a tener en su tramo medio (Sam6 y Sam4) 167 y 265 individuos respectivamente, posteriormente el valor se recupera pero no vuelve a alcanzar las elevadas abundancias del tramo de nacimiento (Sam2 con 1070 individuos). La estación Sam6 recoge la influencia de la urbanización "El Retamar" y principalmente el impacto del embalse de Navacerrada, que produce una reducción del caudal y un régimen artificial de caudales que origina la desaparición de las especies más reófilas y la reducción de la abundancia de las poblaciones. En cuanto a la diversidad encontramos valores moderados con mínimos en cabecera, donde dominan unos pocos taxones con elevadísimas abundancias. Los bajos valores del índice de diversidad se relacionan con la fuerte dominancia, pobre estructura y organización de estas comunidades.

En las estaciones de cabecera (Sam10 y Sam9) la comunidad bentónica está dominada por Baetidae y Simuliidae, aunque en Sam9, además, les acompaña Naididae. Aguas abajo del embalse de Navacerrada (Sam6), que supone una ruptura y tiene un fuerte impacto en las comunidades, aumenta el porcentaje de Lumbriculidae y Chironomidae, que sustituye a Simuliidae; el resto de los grupos desaparecen (como Plecoptera, afectado negativamente por la presencia del embalse) o carecen de relevancia. En la estación Sam4, el río manifiesta una cierta recuperación, ya que a pesar de la dominancia de Leptophlebiidae, los Chironomidae disminuyen hasta casi desaparecer, y aparecen una gran variedad de grupos aunque con bajas abundancias poblacionales, tales como Hidrophilidae, Ancylidae, Physidae, Erpobdellidae, Hydracarina y Ostracoda, todos ellos, sin embargo, desaparecen en la siguiente estación antes de la desembocadura en el embalse de Santillana (Sam2).

Sus afluentes, en comparación, sufren un menor grado de alteración. Los valores de riqueza son altos, 26 taxones/estación en el arroyo Gargantilla y 30 en el Angostura. Los órdenes que más contribuyen a esta riqueza son, en el arroyo Gargantilla: Diptera, Coleoptera, Heteroptera y Ephemeroptera; y en el Angostura: Diptera, Coleoptera, Trichoptera, Ephemeroptera y Heteroptera. La abundancia de macroinvertebrados es muy superior en el Gargantilla (4285 individuos) que en el Angostura (1161 individuos), lo que origina una fuerte dominancia de unos pocos grupos e inferiores valores de diversidad. Ambos arroyos muestran un patrón similar de dominancia por Ephemeroptera $(59 \%$ y $51 \%$ respectivamente), principalmente Baetidae.

\section{Evaluación biológica de la calidad de las aguas}

Los valores del índice IBMWP y las clases de calidad obtenidas se representan en la figura 5. Se trata de ríos de montaña mediterránea silícea, "tipo 11" a los que les correspondería para las condiciones de referencia un valor de 180 de este indicador, los límites de cambio vienen fijados por los valores de EQR 0.78; 0.59; 0.39 y 0.20 respectivamente (BOE 229 de 2008), por lo que encontramos desde aguas de muy buena calidad a deficientes o de mala calidad.

Las localidades situadas en el interior del parque presentan aguas de muy buena calidad (en 


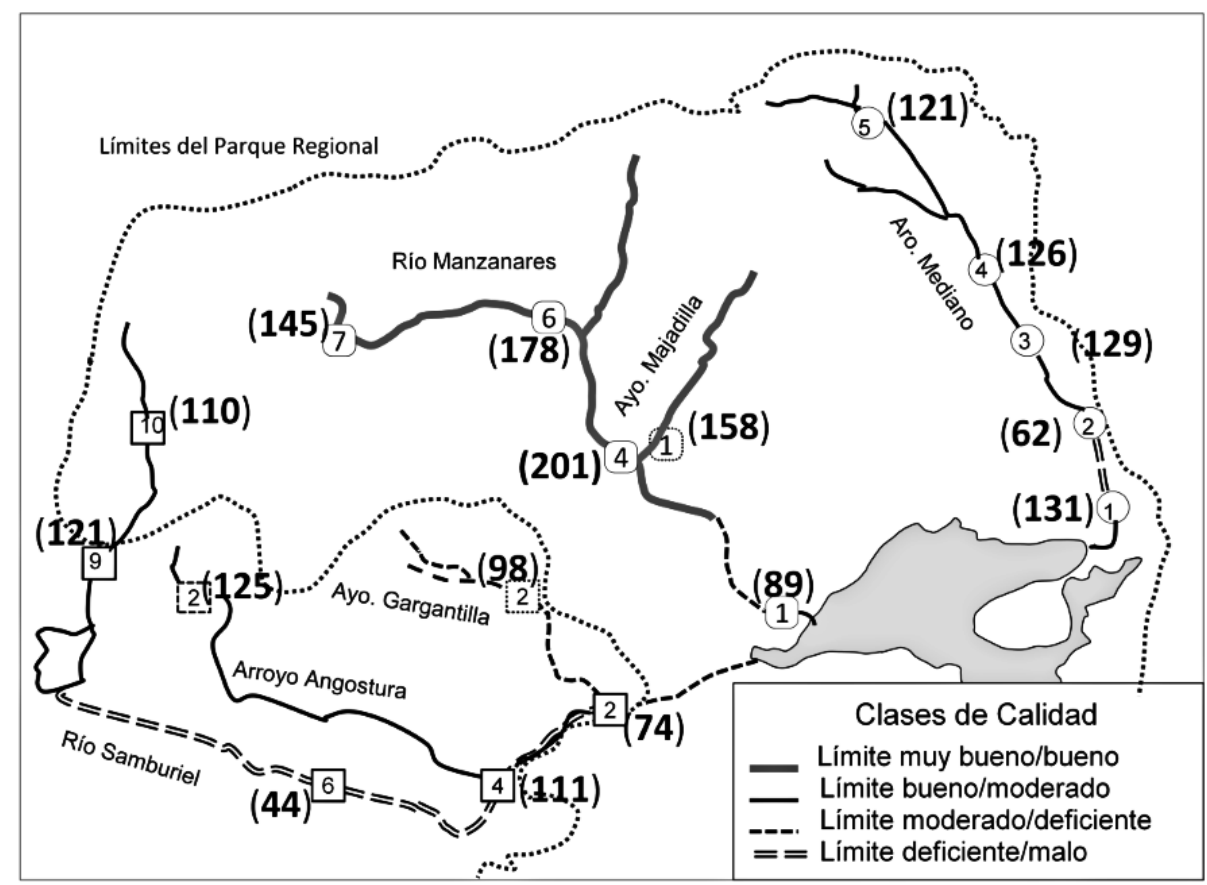

Figura 5. Mapa de la calidad del agua de la cuenca alta del río Manzanares según el índice biológico IBMWP. Map of the water quality in the streams of Manzanares upper basin according to the IBMWP index.

algún caso, por encima del valor considerado de referencia) excepto en las zonas adyacentes a núcleos de población. Así, el río Manzanares, con su arroyo Majadilla, parecen gozar de la mayor calidad, a pesar de la enorme presión recreativa que recibe de las pequeñas represas que mantiene en su tramo alto y de la heredada ocupación del espacio fluvial de sus riberas por construc-

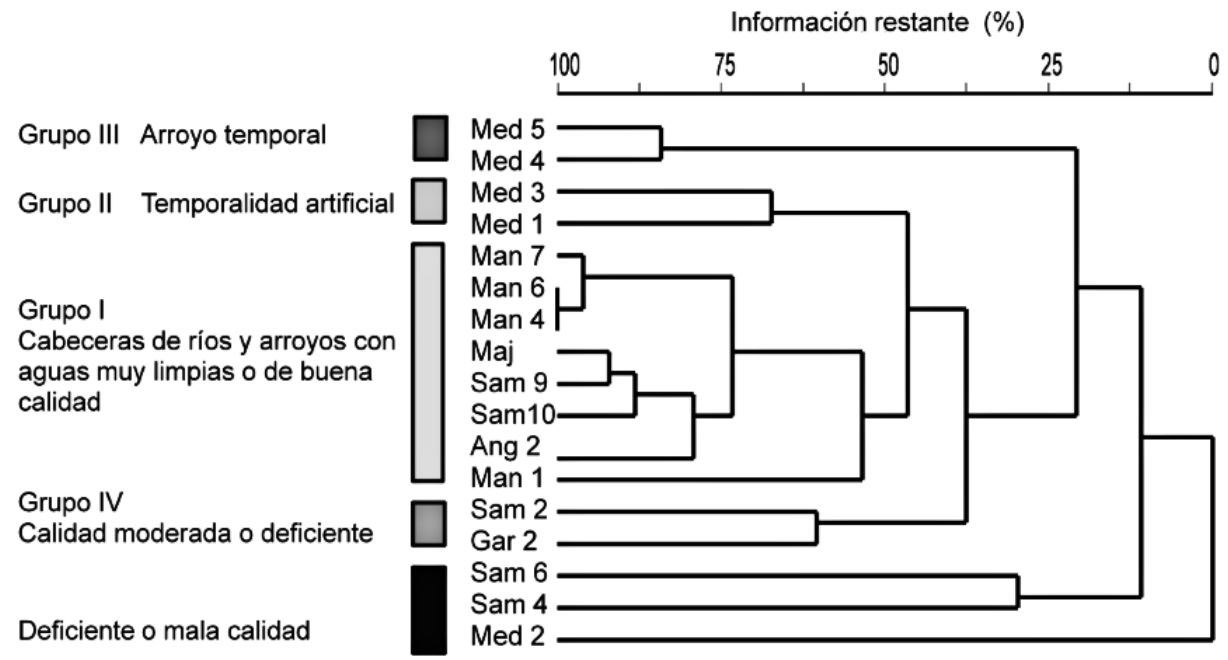

Figura 6. Clasificación de ríos y arroyos Dendrograma de la agrupación de los ríos y arroyos de la cuenca alta del río Manzanares según la similaridad de su fauna de macroinvertebrados. (Análisis de clasificación UPGMA). Cluster analysis of streams in the Manzanares stream upper basin according to the similarity of its benthic macroinvertebrate fauna. (cluster analysis UPGMA). 
ciones residenciales y de servicios hosteleros en su tramo medio y bajo. Únicamente encontramos un evidente empeoramiento de la calidad en la zona fluvial urbana de Manzanares El Real donde, en algunos tramos está semicanalizado. En menor medida, también el arroyo Mediano goza de aguas de buena calidad hasta la derivación de su caudal hacia el embalse de Los Palancares (Med2), aunque mejora en su tramo final.

También presenta aguas de buena calidad el arroyo Angostura (a pesar de no estar incluido dentro del parque) y el nacimiento del río Samburiel hasta la presa de Navacerrada. A partir de aquí el río Samburiel, junto con su arroyo Gargantilla, es el que presenta la peor calidad de todas las corrientes de agua estudiadas. Ambos situados fuera de los límites del PRCAM. Sin duda, esto se relaciona con el número e intensidad de usos más o menos agresivos que convergen en esta cuenca y en el propio cauce. Desde casi su nacimiento, encontramos una importante regulación de caudales por las pequeñas presas localizadas en el valle de La Barranca y, posteriormente, el embalse de Navacerrada, además de la ocupación de una gran extensión de su territorio por áreas urbanas y núcleos residenciales.

\section{Clasificación y ordenación de las comunidades de macroinvertebrados}

Los resultados obtenidos del análisis de agrupación se representan en la figura 6 . Como se puede observar a un nivel de afinidad faunística elevada, al 0.55 ( $55 \%$ de similaridad), el dendrograma muestra 4 grupos diferenciados y 3 estaciones que, por su baja similitud con otras, han quedado sin agrupar.

La principal agrupación está formada por las estaciones de cabecera de ríos y arroyos de mayor caudal, con aguas muy limpias o de buena calidad, localizadas en el interior o en los límites del parque (Man7, Man6 y Man4, Maj1, Sam10, Sam9 y Ang2). Presentan una elevada riqueza específica (entre 21 y 37 taxones distintos) y un número elevado de individuos. Sus comunidades son las más diversificadas, con especies indicadoras de muy buena calidad del agua, aunque puede observarse una dominancia clara de efemerópte- ros. Este primer grupo queda completado con la estación Man1 en la que, si bien disminuye la riqueza y abundancia, la diversidad es elevada y la composición de la comunidad muy semejante.

Posteriormente, se une un segundo grupo formado por las estaciones Med3 y Med1 que, aunque con aguas de buena calidad, presentan una disminución drástica de los valores de abundancia, aunque no en los de riqueza y diversidad.

Las estaciones de nacimiento de este pequeño arroyo (Med5 y Med4) quedan segregadas en un tercer grupo que se une al mayoritario con un menor grado de afinidad. Son tramos temporales de aguas limpias y elevada calidad ambiental, pero con bajísima abundancia de macroinvertebrados y una marcada dominancia de coleópteros leníticos y dípteros, posiblemente debido a su pequeño tamaño y elevada estacionalidad.

Otra agrupación está formada por el tramo bajo del río Samburiel (Sam2) y su arroyo Gargantilla (Gar2), con un nivel muy inferior de similitud entre ellas. Son estaciones localizadas fuera de los límites del parque que presentan una calidad del agua moderada o deficiente, con comunidades más simplificadas con una clara codominancia de efemerópteros y dípteros, siendo importantes los coleópteros en el arroyo y los oligoquetos en el río.

Finalmente, aparecen las tres estaciones más degradadas de todas las cuencas con mala calidad del agua, las del tramo medio del Samburiel (Sam6 y Sam4) y la estación Med2 del arroyo Mediano. Las dos primeras, fuera del PRCAM aguas abajo del embalse de Navacerrada y afectadas además por las urbanizaciones y áreas recreativas de sus riberas; Med2, aunque dentro del PRCAM, justo en su límite oriental afectado por la población de Soto del Real y sus fincas ganaderas.

En resumen, podemos apreciar dos criterios clave de agrupación de estaciones, la contaminación o grado de alteración y la temporalidad, tanto natural como por regulación.

Los resultados del análisis de ordenación multidimensional no paramétrica de las estaciones de muestreo realizado con la matriz de datos de abundancia de las 16 familias más representativas se presentan en la figura 7. Concuerdan con los grupos de estaciones obtenidos anteriormente pero pone de relieve las diferencias existentes 


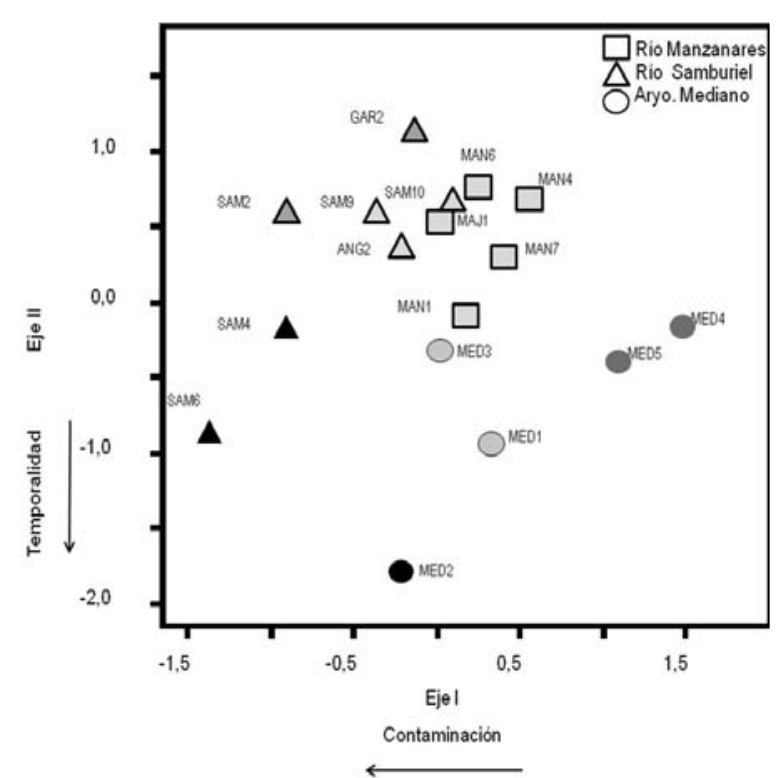

Figura 7. Gráfico de ordenación multidimensional no paramétrica de las estaciones de muestreo en función de las comunidades de macroinvertebrados. Non-metric multidimensional scaling analysis ordination plot based on macroinvertebrate communities.

entre las tres cuencas en cuanto a similitud en la complejidad y conservación de sus comunidades de macroinvertebrados, siendo de entre todos los ríos y arroyos considerados, el Samburiel (excepto su tramo de nacimiento), el más dispar.

En la zona central de ambos ejes se ubican las estaciones del río Manzanares junto con las de cabecera del río Samburiel y la estación Med3 del arroyo Mediano, muy similar a las anteriores excepto por una menor abundancia de organismos. En la parte inferior derecha de la figura se localizan las estaciones del arroyo Mediano, caracterizadas por sus bajos caudales y la alta estacionalidad de los mismos, tanto natural en los tramos de cabecera, como artificial en su tramo bajo. La estación Med2 se encuentra bastante alejada del resto de las estaciones de su cuenca, reflejando con ello las malas condiciones fluviales y la pobreza de sus comunidades, con valores bajos de riqueza, abundancia, diversidad e índice de calidad del agua. En la parte izquierda aparecen las estaciones de la cuenca del Samburiel. Todas ellas se encuentran fuera de los límites del parque y son las que presentan peores condiciones, con las comunidades más simplificadas, menor rique- za e inferiores valores de la mayor parte de los índices. El punto más alejado del núcleo central corresponde al Sam6, tras el embalse de Navacerrada.

El eje I parece representar un gradiente de contaminación, localizándose en su parte negativa las estaciones más alteradas y de peor calidad (todas las del Samburiel, excepto las dos de nacimiento, y el Med2). El eje II representa un gradiente de temporalidad, localizándose en su extremo negativo las estaciones del pequeño arroyo Mediano y las del Samburiel, aguas abajo del embalse de Navacerrada, frente a las estaciones de su parte positiva con aguas permanentes.

\section{DISCUSIÓN}

Según Europarc-España (2010), en nuestro país, el $12.1 \%$ de la superficie terrestre y cerca del $1 \%$ de la superficie marina está protegida con fines de conservación de la naturaleza y, si se incluye la Red Natura 2000, esta cifra alcanza el $28 \%$ del territorio. Pero al igual que en el resto del mundo, no todos los ecosistemas están protegidos adecuadamente (Rodrigues et al., 2004), los ecosistemas acuáticos continentales o limnosistemas han recibido menos atención que cualquier otro ecosistema natural (Abell, 2002), y los arroyos y ríos quizás aún menos (Allan \& Flecker, 1993; Abell et al., 2007).

En nuestro país, cerca del $70 \%$ de los humedales, el $63 \%$ de las marismas y el $14.3 \%$ de la red de ríos y arroyos tienen alguna designación como espacio natural protegido (EuroparcEspaña, 2010) aunque la gran mayoría de estas figuras presentan una insuficiente definición de los valores por los que se declaran las áreas protegidas y sus objetivos de gestión. Además, pocas de estas áreas han sido creadas, diseñadas y gestionadas específicamente para proteger los limnosistemas (Pringle, 2001; Saunder et al., 2002; Collares-Pereira \& Cowx, 2004), y la mayoría están comúnmente protegidos solo incidentalmente como parte de su inclusión en reservas terrestres (Lake, 1980; Skelton et al., 1995; Saunder et al., 2002; Locke \& Dearden, 2005; Nel et al., 2007) por lo que no siempre aseguran la calidad biológica y conservación de la biodiversi- 
dad (Mancini et al., 2005) ni de los sistemas fluviales y los importantes servicios que estos proporcionan (Abel et al., 2007).

El PRCAM también presenta estas limitaciones ya que en origen fue creado alrededor del enclave de La Pedriza y sus valores geomorfológicos, y para regular las actividades y la demanda de uso para esparcimiento recreativo de aficionados de la ciudad de Madrid a la montaña, al que se veía sometido y, aunque posteriormente ha sido ampliado y recalificado sucesivas veces, los ecosistemas acuáticos no han tenido una gran relevancia a pesar de que la red hidrográfica de la cabecera del río Manzanares se localiza dentro de su ámbito, da nombre al parque y vertebra su territorio. En general, se trata de cauces pequeños y medianos con pendientes elevadas cuyos lechos están constituidos por rocas de gran tamaño, cantos rodados, gravas y arenas gruesas, que aunque en zonas urbanizadas, suelen estar canalizados. Son relativamente impermeables y presentan una rápida respuesta a episodios tormentosos, con rápidas crecidas y descensos de caudal, mostrando una alta temporalidad como corrobora el hecho de que el $45.17 \%$ de los cursos de agua se encontraban secos o únicamente presentaban someras charcas aisladas en el momento del muestreo.

Presentan aguas dulces de muy baja mineralización, las menos mineralizadas y de mejor calidad se sitúan dentro del parque, existiendo un aumento de la mineralización no solo en los tramos más bajos de estos ríos, sino también en los ríos y tramos de cabecera fuera del parque, por lo que este aumento puede reflejar el inicio de un uso más intensivo de las cuencas de drenaje y riberas fluviales. Además, también cambia su composición de bicarbonatado sódica a clorurado cálcica tras la presencia de poblaciones y embalses. El predominio del sodio en los tramos de mayor altitud refleja indirectamente la litología impermeable de la zona y los aportes de agua meteórica. Según Clenaghan et al. (1998), localidades bajo la influencia de la agricultura tienen típicamente más nutrientes, cloruros, $\mathrm{pH}$ y variables relacionadas como alcalinidad y dureza. En nuestro caso, el inesperado y brusco aumento de los cloruros en los tramos medio y final del río Samburiel, si consideramos las escasas fuentes naturales de cloruros derivadas de la geología y suelos de la zona, evidencia la incorporación de vertidos difusos y contaminantes al agua circulante por estos tramos y sus afluentes Angostura y Gargantilla, los tres situados fuera del PRCAM y con núcleos de población y zonas residenciales en sus inmediaciones.

Las concentraciones de los principales nutrientes en el agua son bajas, especialmente el nitrógeno que sólo aumenta a la salida del embalse de Navacerrada, tras la retención del agua en dicho embalse y la disminución drástica del caudal circulante por este tramo. Sin embargo, se detectan valores inesperadamente elevados y descompensados de fosfatos en la desembocadura del río Manzanares, en su arroyo Majadilla y en el arroyo Mediano, todos ellos dentro del parque. Esta entrada de fósforo al sistema no puede ser de origen edáfico, debido al tipo de suelos y litología de estas cuencas (rocas silíceas muy antiguas), por lo que podría ser consecuencia del tipo de actividades humanas a las que están sometidos, principalmente actividades recreativas tanto de senderismo como en sus propias riberas y cauce (incluido el baño). En el arroyo Mediano es importante además la detracción de caudal y la influencia de las áreas ganaderas de Soto del Real.

Las comunidades de macroinvertebrados de las tres cuencas fluviales difieren entre sí en composición y riqueza taxonómica, abundancia, diversidad, complejidad y estado de conservación. Diferencias muy ligadas a dos tipos de perturbaciones, básicamente la contaminación y la temporalidad, tanto natural en cabeceras y arroyos como artificial por detracción del caudal (Med2) o regulación por embalses (Sam6 y Sam4). La contaminación y la regulación son dos de las principales amenazas de la integridad biótica de la mayor parte de los ríos del mundo (SzöllsiNagy et al., 1998; Malmqvist \& Rundle, 2002; Saunders et al., 2002) y de los ríos mediterráneos (Prenda et al., 2006). La intensidad de ambos tipos de alteraciones está ligada a la figura de protección del Parque Regional, por lo que aparentemente parece contribuir a la conservación de los ecosistemas fluviales incluidos en el mismo. Encontramos tres tipos de situaciones: las localidades situadas en el interior del parque, en el núcleo central del área protegida (río Manzanares 
y arroyo Majadilla) que presentan la mejor calidad biótica con comunidades de elevada riqueza taxonómica, bien estructuradas y complejas, y con un tipo de composición faunística semejante a los ríos del N-W y centro peninsular, en los que domina la riqueza de tricópteros con efemerópteros como acompañante y, en los pequeños cursos plecópteros como segundo acompañante (AlbaTercedor et al., 1992), por lo que podemos pensar que sus comunidades no se hallan modificadas. Incluso en alguna de estas estaciones se supera el valor IBMWP establecidos para estaciones de referencia de esta tipología fluvial. Las estaciones situadas dentro del PRCAM pero en las zonas limítrofes del área formalmente protegida (nacimiento del río Samburiel y el arroyo Mediano excepto Med2) o en la zona externa adyacente (arroyo Angostura) acusan un moderado empobrecimiento y descenso en su calidad biótica. Están afectadas principalmente por ganadería extensiva y actividades recreativas, aunque empieza a ser preocupante la creciente proliferación de construcciones aisladas en estos territorios. Finalmente, las que se encuentran fuera de los límites del PRCAM, caso del río Samburiel y su arroyo Gargantilla y el tramo medio-bajo del arroyo Mediano (a pesar de su ubicación en el interior del área protegida) son las que presentan condiciones deficientes o malas, con comunidades muy simplificadas y empobrecidas. Estos ríos presentan alterados sus regímenes hidrológicos por embalses o derivación de caudal y el hábitat físico por canalización, lo que suele coincidir con proximidad a urbanizaciones, poblaciones y carreteras, que habitualmente conlleva la destrucción de sus riberas por la tala y movimiento de tierras. Todas ellas, actuaciones que han aumentado de escala e impacto en la última centuria (Malmqvist \& Rundle, 2002; Nilson et al., 2005).

Estos resultados concuerdan con los obtenidos por otros autores (Rieradevall et al., 1999; Bonada et al., 2000; Mancini et al., 2005; Thieme et al., 2005; Nel et al., 2007) y ponen de manifiesto la utilidad y necesidad de protección de los espacios naturales a fin de preservar también la diversidad y buen estado ecológico de nuestros cursos de agua (Prenda et al., 2006). Pero el efecto beneficioso del PRCAM no se debe a un buen diseño y gestión del área protegida en relación a los limnosistemas, que tradicionalmente han sido ignorados en cuanto a objetivos, propuestas y estrategias de evaluación y gestión, sino a la localización de las explotaciones y actividades humanas más agresivas en las zonas externas del parque. Como ocurre en otros casos, las áreas protegidas no protegen adecuadamente pero hay una marcada mejora en la integridad de los ríos dentro de estas áreas comparadas con las de fuera del área (Nel et al., 2007).

El único sistema fluvial que podríamos considerar no alterado es la subcuenca de nacimiento del río Manzanares hasta su tramo medio, enclavado en el corazón de La Pedriza, núcleo central del parque y que constituye la parte más inaccesible, donde no está permitido el tráfico rodado y la afluencia de visitantes es sensiblemente menor debido a la dificultad de los senderos y vías de acceso. Todos los demás sistemas fluviales de la cuenca, tanto dentro como fuera del PRCAM, se encuentran en la actualidad más o menos alterados y con una degradación de su calidad biológica desde moderada a crítica dependiendo del número y tipo de usos por los que están afectados.

Esta alarmante situación motiva la necesidad de contemplar estrategias específicas de protección y conservación de los ecosistemas acuáticos continentales centradas en conservar procesos a la vez bióticos y abióticos, mantener la conectividad fluvial y la naturalidad de los procesos hidrológicos (Pringle, 2001, Baron et al., 2002, Silk \& Ciruna, 2004; Locke \& Dearden, 2005). Las áreas protegidas y la gestión integrada de la cuenca son complementarias, y esta última debe ser la organizadora de los principios de conservación de los limnosistemas, con las áreas protegidas sirviendo como un componente estratégico (Abel et al., 2007). De acuerdo con este autor, la adopción de una estrategia de protección jerárquica puede ser una buena solución al cada vez más inalcanzable objetivo de proteger cuencas completas. Gilmar et al. (2004) han encontrado que incluso la planificación de la conservación de la biodiversidad acuática está mal representada en la mayoría de planes de gestión integrada de cuencas fluviales (IRBM), especialmente en países desarrollados. Adoptan- 
do esta estrategia se delimitarían tres zonas en el PRCAM: el núcleo del parque donde se ubican los ríos bien conservados se definiría como área focal (freshwater focal area) que requiere protección y por tanto restricciones severas para su mantenimiento; las áreas limítrofes del parque, con ríos moderadamente alterados como zona de gestión crítica (critical management zone) en las que se pueden definir unas ciertas restricciones de uso, incluso únicamente de forma temporal en los momentos más críticos pero sin exclusión de todo tipo de usos se podrían mantener los usos ganaderos y recreativos aunque evidentemente regulados; $y$, finalmente, el tercer grupo de ríos, los que se encuentran completamente degradados, localizados fuera de los límites del PRCAM y el tramo del Mediano afectado por la extracción de caudal, que requieren una gestión activa (catchment management zone) con prácticas de manejo para minimizar los impactos de la regulación o el tratamiento de todo el agua residual; y prácticas de restauración, como es el caso de la cubierta vegetal riparia, actualmente casi inexistente o con plantaciones de chopos y especies exóticas en lugar de las fresnedas y saucedas naturales, o de los tramos canalizados.

Coincidiendo con este autor, el diseño y gestión efectiva de áreas de protección acuática continental requerirá que las ideas más innovadoras en biología de la conservación, biología y ecología de las aguas continentales, planificación del territorio, hidrología, economía ambiental y otras ciencias sociales vuelvan a estar unidas. Combinando estas disciplinas se pueden empezar a tender puentes entre divisiones artificiales como planificación acuática continental, terrestre y marina, especialmente cuando los procesos hidrológicos se reconocen como centrales en la planificación y gestión (Abel et al., 2007).

\section{AGRADECIMIENTOS}

Agradecemos a la dirección y personal del PRCAM las facilidades de acceso prestadas para la realización del trabajo de campo y su cordial disposición. Este trabajo ha sido financiado por el Proyecto de Investigación de la Comunidad de Madrid "Valoración y propuesta de recuperación de la Diversidad Biológica de los ecosistemas fluviales de la cuenca Alta del Manzanares (Madrid)" (ref. 07M/0060/2000). También deseamos agradecer a dos revisores anónimos sus útiles comentarios y las sugerencias aportadas, que han enriquecido el manuscrito.

\section{REFERENCIAS}

ABELL, R. 2002. Conservation biology for the biodiversity crisis: a freshwater follow-up. Conservation Biology, 16: 1435-1437.

ABELL, R., J. D. ALLAN \& B. LEHNER. 2007. Unlocking the potential of protected areas for freshwaters. Biological Conservation, 134: 48-63.

ALBA-TERCEDOR, J. G. GONZÁLEZ \& M. A. PUIG. 1992. Present level of knowledge regarding fluvial macroinvertebrates communities in Spain. Limnetica, 8: 231-241.

ALBA-TERCEDOR, J., P. JÁIMEZ-CUÉLLAR, M. ÁLVAREZ, J. AVILÉS, N. BONADA, J. CASAS, A. MELLADO, M. ORTEGA, I. PARDO, N. PRAT, M. RIERADEVALL, S. ROBLES, C. E. SÁINZ-CANTERO, A. SÁNCHEZ-ORTEGA, M. L. SUÁREZ, M. TORO, M. R. VIDAL-ABARCA, S. VIVAS \& C. ZAMORA-MUÑOZ. 2002. Caracterización del estado ecológico de ríos mediterráneos ibéricos mediante el índice IBMWP (antes BMWP'). Limnetica, 21 (3-4): 175-185.

ALLAN, J. D. \& A. S. FLECKER. 1993. Biodiversity conservation in running waters. Bioscience, 43: 32-43.

ALONSO, J. J. \& J. G. CATALAN. 1967. Estudio de microscopía electrónica y químico de las aguas de los ríos Jarama, Guadalix, Lozoya y Manzanares. Documentos de investigación hidrológica, 1: 25-35.

BARON, J. S., N. L. POFF, P. L. ANGERMEIER, C. N. DAHM, P. H. GLEICK, N. G. HAIRSTON, R. B. JACKSON, C. A. JOHNSTON, B. D. RICHTER \& A. D. STEINMAN. 2002. Meeting ecological and societal needs for freshwater. Ecological Applications, 12: 1247-1260.

BENETTI, C. J., A. I. ALONSO \& J. GARRIDO. 2007. Comparación de la comunidad de coleópteros acuáticos (Adephaga y Polyphaga) en dos cuencas hidrográficas con distinto grado de acción 
antropogénica (Pontevedra, NO de España). Limnetica, 26(1): 115-128.

BLANCO, E., M. A. CASADO, M. COSTA, R. ESCRIBANO, M. GARCÍA ANTÓN, M. GÉNOVA, A. GÓMEZ MANZANEQUE, F. GÓMEZ MANZANEQUE, J. C. MORENO, C, MORLA, P. REGATO \& H. SAINZ OLLERO. 1997. Los bosques ibéricos. Una interpretación geobotánica. Planeta. Barcelona. 572 pp.

BONADA, N., M. RIERADEVALL \& N. PRAT. 2000. Temporalidad y contaminación como claves para interpretar la biodiversidad de macroinvertebrados en un arroyo mediterráneo (Riera de Sant Cugat, Barcelona). Limnetica, 18: 81-90.

BONADA, N., N. PRAT, A. MUNNÉ, M. RIERADEVALL, J. ALBA-TERCEDOR, M. ÁLVAREZ, J. AVILÉS, J. CASAS, P. JÁIMEZ-CUÉLLAR, A. MELLADO, G. MOYÁ, I. PARDO, S. ROBLES, G. RAMON, M.L. SUÁREZ, M. TORO, M.R. VIDAL-ABARCA, S. VIVAS \& C. ZAMORAMUÑOZ. 2002. Ensayo de una tipología de las cuencas mediterráneas del proyecto GUADALMED siguiendo las directrices de la Directiva Marco del Agua. Limnetica, 21(3-4): 77-98.

BONADA, N., M. RIERADEVALL \& N. PRAT. 2007. Macroinvertebrate community structure and biological traits related to flow permanence in a Mediterranean river network. Hydrobiologia, 589: 91-106.

CASAS, J., M. O. GESSNER, P. H. LANGTON, D. CALLE, E. DESCALS \& M. J. SALINAS. 2006. Diversity of patterns and processes in rivers of eastern Andalucía. Limnetica, 25(1-2): 155-170.

COLLARES-PEREIRA, M. J. \& I. G. COWX. 2004. The role of catchment scale environmental management in freshwater fish conservation. Fisheries Management and Ecology, 11: 303-312.

CUBILLO, F. 1985. Plan Integral del Agua en Madrid. Calidad de aguas superficiales. Campañas intensivas de control en ríos. Dirección General de Recursos Hidráulicos. Publ. no 10. Comunidad de Madrid. 230 pp.

CUBILLO, F., C. CASADO \& V. CASTILLO. 1990. Caudales ecológicos. Estudio de regímenes de caudales mínimos en los cauces de la Comunidad de Madrid. Agencia de Medio Ambiente. Comunidad de Madrid. 305 pp.

CLENAGHAM, C., J. O'HALLORAN, P. S. GILLER \& N. ROCHE. 1998. Longitudinal and temporal variation in the hydrochemistry of streams in an
Irish conifer afforested catchment. Hydrobiologia, 389: 63-71.

EUGSTER, H. P. \& L. A. HARDIE. 1987. Saline Lakes. In: Lakes: Chemistry, Geology, Physics. 237293. A. Lerman (ed.). Springer Verlag.

EUROPARC-ESPAÑA. 2010. Anuario Europarc-España del estado de los espacios naturales protegidos 2009. Ed. FUNGOBE. Madrid. 104 pp.

GARCÍA DE JALÓN, D. \& M. GÓNZALEZ DEL TÁNAGO. 1983. Estudio biotipológico de las comunidades piscícolas de la Cuenca del Duero. Voletín de la Estación Central de Ecolgía, 12(24): 57-66.

GILMAN, R. T., R. A. ABELL \& C. E. WILLIAMS. 2004. How can conservation biology inform the practice of Integrated River Basin Management? International Journal of River Basin Management, 2(2):1-14

GÓMEZ-LIMÓN, F. J. \& J. GARCÍA-AVILÉS. 1992. Estudio del impacto de las actividades recreativas en dos cauces fluviales del Parque Regional de la Cuenca Alta del Manzanares (área recreativa de la Pedriza). Publ. Centro de Investigación "Fernando González Bernáldez". 23 pp.

GONZÁLEZ DEL TÁNAGO, M. \& D. GARCÍA DE JALÓN. 2006. Propuesta de caracterización jerárquica de los ríos españoles para su clasificación según la Directiva Marco de la Unión Europea. Limnetica, 25(3): 693-712.

GUTIÉRREZ TEIRA, B. 2003. Variaciones de las comunidades y poblaciones de macroinvertebrados del tramo alto del río Manzanares a causa de las temperaturas. Posibles efectos del cambio climático. Tesis Doctoral. Universidad Politécnica de Madrid. Escuela Técnica Superior de Ingenieros de Montes. 360 pp.

LAKE, P. S. 1980. Conservation. In: An ecological basis for water resource management. W. D.Williams (ed.): 163-173. Australian National University Press, Camberra.

LESKA, S. F., J. R. KARR \& R. W. WISSEMAN, 1996 Assesing invertebrate responses to human activities: evaluating alternatives approaches. $J . N$. Am. Benthol. Soc., 15: 212-231.

LOCKE, H. \& P. DEARDEN. 2005. Rethinking protected area categories and the new paradigm. Environmental Conservation, 32:1-10

LOSADA, L. 2004. Estructura de las comunidades bentónicas y calidad del agua en los ríos de la Cuenca Alta del Manzanares. D. E. A., Universidad Autónoma de Madrid. 81 pp. 
MALMQVIST, B. \& R. RUNDLE. 2002. Threats to the running water ecosystems of the world. Environmental Conservation, 29:134-153.

MANCINI, L., P. FORMICHETTI, A. ANSELMO, L. TANCIONI, S. MARCHINI \& A. SORACE. 2005. Biological quality of running waters in protected areas: the influence of size and land use. Biodiversity and Conservation, 14: 351-364.

MARTÍNEZ-BASTIDA, J. J., M. ARAÚZO \& M. VALLADOLID. 2006. Diagnóstico de la calidad ambiental del río Oja (La Rioja, España) mediante el análisis de la comunidad de macroinvertebrados bentónicos. Limnetica, 25(3): 733-744.

MONTES, C., A. LLORCA, A. STERLING, C. CASADO, I. CASTRO, A. MONZÓN \& L. SANTAMARÍA. 1987. Directrices para la recuperación ecológica del tramo medio del río Manzanares. Canal de Isabel II. Comunidad de Madrid. 142 pp.

MONZÓN, A. 1986. Organización longitudinal de comunidades de macroinvertebrados acuáticos en una cuenca del sistema Central (Río Manzanares) y su relación con algunas variables funcionales. Tesis de Licenciatura. Universidad Autónoma de Madrid. Madrid. 119 pp.

MONZÓN, A., C. CASADO, C. MONTES \& D. GARCÍA DE JALÓN. 1991. Organización funcional de las comunidades de macroinvertebrados acuáticos de un sistema fluvial de montaña (Sistema Central, río Manzanares, España). Limnetica, 7: 97-112

NEL, J. L., D. J. ROUX, G. MAREE, C. J. KLEYNHANS, J. MOOLMAN, B. REYERS, M. ROUGET \& R. M. COWLING. 2007. Rivers in peril and outside protected areas: a systematic approach to conservation assessment of river ecosystems. Diversity Distribution, 13: 341-352.

NILSON, C., C. A. REIDY, M. DYNESIUS \& C. REVENGA. 2005. Fragmentation and flow regulation of the world's large river systems. Science, 308: 405-408.

ORTIZ, J. D. \& M. A. PUIG. 2007. Point source effects on density, biomass and diversity of benthic macroinvertebrates in a mediterranean stream. $R i$ vers Research and Applications, 23: 155-170.

OSCOZ, J., F. CAMPOS \& M. C. ESCALA. 2006. Variación de la comunidad de macroinvertebrados bentónicos en relación con la calidad de las aguas. Limnetica, 25(3): 683-692.

PÉREZ SILES, B. 2004. Dinámica poblacional y producción secundaria de Epeorus torrentium (Clase Insecta, Orden Ephemeroptera) en un río medi- terráneo de alta montaña. Tesis de LicenciaturaDiploma de Estudios Avanzados. Universidad Autónoma de Madrid, 47 pp.

PINEDA, F. D., F. G. DI CASTRI, C. ORCOYEN \& J. R. VILLANUEVA. 1991. Estudio y conservación de la diversidad biológica. En: Diversidad Biológica/Biological Diversity. F. Pineda et al., (eds.): 15-19. Fundació Areces-ADENA-WWF/SCOPE. Madrid.

POOLE, G. C. 2010. The importance of channel network topology and dynamic. J. N. Am. Benthol. Soc., 29 (1): 12-25.

PRENDA, J., M. CLAVERO, F. BLANCO-GARRIDO, A. MENOR \& V. HERMOSO. 2006. Threats to the conservation of biotic integrity in Iberian fluvial ecosystem. Limnetica, 25(1-2): 377-388.

PRINGLE, C. M. 2001. Hydrologic connentivity and the management of biological reserves: A global perspective. Ecological Applications, 11: 981-998.

RIERADEVALL, M., N. BONADA \& N. PRAT. 1999. Community structure and water quality in the mediterranean streams of a natural parck (St. Llorenç del Munt, EN Spain). Limnetica, 17: 4556.

RODRIGUES, A. S. L., S. J. ANDELMAN, M. I. BAKARR, L. BOITANI, T. M. BROOKS, R. M. COWLING, L. D. C. FISHPOOL, G. A. B. FONSECA, K. J. GASTON, M. HOFFMANN, J. S. LONG, P. A. MARQUET, J. D. PILGRIM, R. J. PRESSEY, J. SCHIPPER, W. SECHREST, S. N. STUART, L. G. UNDERHILL, R. W. WALLER, M. E. J. WATTS \& X. YAN. 2004. Effectiveness of the global protected area network in representin species diversity. Nature, 428: 640-643.

SAUNDERS, D. L., J. J. MEEUWIG \& A. C. J. VINCENT. 2002. Freshwater protected areas: Strategies for conservation. Conservation Biology, 16(1): 30-41.

SERRANO, A. 2000. Caracterización espacio-temporal de las comunidades de cianobacterias epilíticas de un arroyo de montaña mediterráneo, el Arroyo Mediano (Comunidad de Madrid). Tesis de Licenciatura. Universidad Autónoma de Madrid. 145 pp.

SERRANO, A., P. MATEO \& E. PERONA. 2004. Estructura y composición de cianobacterias bentónicas de un arroyo de montaña mediterráneo, el arroyo Mediano (Madrid). Limnetica, 23(1-2): 83-94.

SKELTON, P. H., J. A. CAMBRAY, A. LOMBARD \& G. A. BENN. 1995. Patterns of distribution and conservation status of freshwater fishes in South 
Africa. South African Journal of Ichthyology, 30: 71-81.

SUTIN, S., M. JAROENSUTASINEE \& K. JAROENSUTASINEE. 2007. Water quality and freshwater fish diversity at Khao Luang National Park, Thailand. World Academy of Science, Engineering and Technology, 36: 28-31.

SZÖLLOSI-NAGY, A., P. NAJLIS \& G. BJÖRKLUND. 1998. Assessing the world's freshwater resources. Nature and Resources, 34(1): 8-18.

THIEME, M. L., R.A. ABELL, A. LEHNER, E. DINERSTEIN, M. L. J. STIASSNY, P. H. SKELTON, G. G. TEUGELS, A. KAMDEM-THOAM \& D. M. OLSON. 2005. Freshwater ecoregions of Africa and Madagascar: a conservation assessment. Island Press, Washington, D.C. 431 pp.

TORRALBA, A. \& F. J. OCHARAN. 2007. Comparación del muestreo de macroinvertebrados bentónicos fluviales con muestreador surber y con red manual en ríos de Aragón (NE Península Ibérica). Limnetica, 26(1): 13-24.

VALLADOLID, M., J. J. MARTÍNEZ-BASTIDA, M. ARAÚZO \& C. GUTIÉRREZ. 2006. Abundancia y biodiversidad de los macroinvertebrados del río Oja (La Rioja, España). Limnetica, 25(3): 745-752. VÉLAZ DE MEDRANO, L. \& J. UGARTE. 1933. Estudio monográfico del río Manzanares. Publ. Inst. For. de Inv. y Exp. $\mathrm{N}^{\mathrm{o}} 11$. Madrid. 68 pp. 